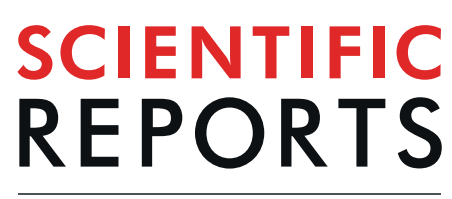

natureresearch

\title{
Prospective diagnostic accuracy study of plasma soluble ST2 for diagnosis of acute aortic syndromes
}

Fulvio Morello $\mathbb{1}^{1,2^{*}}$, Alice Bartalucci ${ }^{1}$, Marco Bironzo ${ }^{1}$, Marco Santoro ${ }^{1}$, Emanuele Pivetta $\mathbb{1}^{1}$, Alice lanniello ${ }^{3}$, Francesca Rumbolo ${ }^{3}$, Giulio Mengozzi $\mathbb{1}^{3}$ \& Enrico Lupia $\mathbb{1}^{1,2}$

Acute aortic syndromes (AASs) are difficult to diagnose emergencies. Plasma soluble ST2 (sST2), a prognostic biomarker for heart failure, has been proposed as a diagnostic biomarker of AASs outperforming D-dimer, the current diagnostic standard. We performed a prospective diagnostic accuracy study of sST2 for AASs in the Emergency Department (ED). In 2017-2018, patients were enrolled if they had $\geq 1$ red-flag symptoms (chest/abdominal/back pain, syncope, perfusion deficit) and a clinical suspicion of AAS. SST2 was detected with the Presage ${ }^{\circledR}$ assay. Adjudication was based on computed tomography angiography (CTA) or on diagnostic outcome inclusive of 30-day follow-up. 297 patients were enrolled, including 88 with AASs. The median age was 67 years. In 162 patients with CTA, the median sST2 level was $41.7 \mathrm{ng} / \mathrm{mL}$ (IOR 29.4-103.2) in AASs and $34.6 \mathrm{ng} / \mathrm{mL}$ (IQR 21.4-51.5) in alternative diagnoses $(P=0.005)$. In ROC analysis, the AUC of sST2 was 0.63 , as compared to 0.82 of D-dimer $(P<0.001)$. Sensitivity and specificity values of sST2 associated with different cutoffs were: $95.5 \%$ and $10.8 \%(\geq 12 \mathrm{ng} / \mathrm{mL}$ ), $84.1 \%$ and $29.7 \%$ ( $\geq 23.7 \mathrm{ng} / \mathrm{mL}$ ), $35.2 \%$ and $85.1 \%(\geq 66.5 \mathrm{ng} / \mathrm{mL})$. Results were similar in the full cohort. In conclusion, in patients from a European ED, plasma sST2 provided modest accuracy for diagnosis of AASs.

Acute aortic syndromes (AASs), which include acute aortic dissection (AAD), intramural aortic hematoma $(\mathrm{IMH})$, penetrating aortic ulcer (PAU) and spontaneous aortic rupture (SAR), are severe cardiovascular emergencies affecting 5-10 per 100.000 individuals a year ${ }^{1}$. A conclusive diagnosis of AASs requires advanced aortic imaging, typically computed tomography angiography (CTA), which uses ionizing radiations and may cause contrast-induced nephropathy and anaphylaxis. However, selection of the right patient to image with CTA is cumbersome, because AASs present with unspecific symptoms such as truncal pain, syncope, neurological deficits and hindlimb ischemia. These are leading causes of Emergency Department (ED) visits worldwide, and most patients with these symptoms are affected by more common conditions, such as acute coronary syndromes, gastrointestinal diseases and muscle-skeletal pain. Hence, the misdiagnosis rate of AASs is high, affecting clinical outcomes $^{2-4}$. Increasing use of CTA in EDs has led to a high number of CTA exams requested for suspected AAS turning out negative, but has not substantially changed misdiagnosis rates, indicating that pre-test patient selection remains a key node ${ }^{5-7}$. Development of reliable diagnostic biomarkers discriminating AASs could positively impact on their diagnostic workup, as for other cardiovascular emergencies such as acute coronary syndromes and pulmonary embolism ${ }^{8,9}$.

So far, potential biomarkers of AASs have been searched for within aortic layers (e.g. smooth muscle myosin, soluble elastin fragments, calponin, CD40 ligand), within inflammation/remodeling pathways (e.g. matrix metalloproteinases) and within coagulation processes mobilized by blood exposure to non-endothelial surfaces (e.g. platelets, D-dimer ${ }^{10}$. Currently, D-dimer is the only biomarker clinically applicable to the workup of AASs ${ }^{11,12}$. Owing to high sensitivity, D-dimer can be used for diagnostic rule-out of AASs, if the pre-test probability is sufficiently low ${ }^{13}$. However, D-dimer can be increased by several conditions such as inflammation, infection, cancer, trauma and age, leading to low diagnostic specificity. On these grounds, the quest for additional diagnostic biomarkers for AASs remains open and vibrant.

ST2 (suppression of tumorigenicity 2, encoded by interleukin 1 receptor-like 1 or IL1RL-1) is a member of the interleukin-1 receptor family, regulating immune-responses ${ }^{14}$. Soluble ST2 (sST2), a truncated form

${ }^{1}$ S.C.U. Medicina d'Urgenza, Molinette Hospital, A.O.U. Città della Salute e della Scienza, Torino, Italy. ${ }^{2}$ Dipartimento di Scienze Mediche, Università degli Studi di Torino, Torino, Italy. ${ }^{3}$ S.C. Biochimica Clinica, A.O.U. Città della Salute e della Scienza, Torino, Italy. *email: fmorello@cittadellasalute.to.it 


\begin{tabular}{|l|c|c|c|c|}
\hline Variable & $\begin{array}{l}\text { Total patients } \\
(\mathbf{n}=\mathbf{2 9 7})\end{array}$ & AASs $(\mathbf{n = 8 8})$ & AltDs (n=209) & $\boldsymbol{P}$-value \\
\hline Female gender & $94(31.6 \%)$ & $26(29.5 \%)$ & $68(32.5 \%)$ & 0.61 \\
\hline Age $[\mathrm{y}]$ & $67(55-78)$ & $72(59.5-80)$ & $65(53-77)$ & 0.04 \\
\hline Hypertension & $176(59.3 \%)$ & $62(70.5 \%)$ & $114(54.5 \%)$ & 0.011 \\
\hline Diabetes & $27(9.1 \%)$ & $7(8 \%)$ & $20(9.6 \%)$ & 0.66 \\
\hline Dyslipidemia & $18(6.1 \%)$ & $3(3.4 \%)$ & $15(7.2 \%)$ & 0.21 \\
\hline Smoking & $87(29.3 \%)$ & $26(29.5 \%)$ & $61(29.2 \%)$ & 0.95 \\
\hline Drug use & $3(1 \%)$ & $2(2.3 \%)$ & $1(0.5 \%)$ & 0.16 \\
\hline Coronary art. dis. & $37(12.5 \%)$ & $6(6.8 \%)$ & $31(14.8 \%)$ & 0.06 \\
\hline Active cancer & $2(0.7 \%)$ & $0(0 \%)$ & $2(1 \%)$ & 0.36 \\
\hline Periph. art. dis. & $1(0.3 \%)$ & $0(0 \%)$ & $1(0.5 \%)$ & 0.52 \\
\hline Abdominal aortic an. & $14(4.7 \%)$ & $5(5.7 \%)$ & $9(4.3 \%)$ & 0.61 \\
\hline Previous AAS & $10(3.4 \%)$ & $4(4.5 \%)$ & $6(2.9 \%)$ & 0.47 \\
\hline Systolic BP $[\mathrm{mmHg}]$ & $140(120-160)$ & $140(110-170)$ & $140(125-160)$ & 0.48 \\
\hline Diastolic BP $[\mathrm{mmHg}]$ & $80(70-90)$ & $80(60-95)$ & $80(75-90)$ & 0.04 \\
\hline Heart rate $[\mathrm{bpm}]$ & $75(68-87)$ & $72(64-83)$ & $75(70-89)$ & 0.15 \\
\hline Time from onset $[\mathrm{h}]$ & $4(2-11)$ & $3(2-6)$ & $5(2-14)$ & 0.02 \\
\hline WBC count $\left[* 10^{3} / \mu \mathrm{L}\right]^{\mathrm{A}}$ & $9.07(7.22-11.71)$ & $11.66(9.1-14.35)$ & $8.14(6.78-10.45)$ & $<0.001$ \\
\hline Creatinine $[\mathrm{mg} / \mathrm{dL}]^{\mathrm{B}}$ & $0.97(0.82-1.16)$ & $1.06(0.86-1.21)$ & $0.93(0.81-1.12)$ & 0.004 \\
\hline Troponin T $[\mathrm{ng} / \mathrm{mL}]^{\mathrm{C}}$ & $11(7-23)$ & $20(10-41)$ & $10(5-18)$ & $<0.001$ \\
\hline D-dimer $[\mathrm{ng} / \mathrm{mL}]^{\mathrm{D}}$ & $780(331.5-3466.5)$ & $6301(1813-28646)$ & $471(265.5-1002.5)$ & $<0.001$ \\
\hline
\end{tabular}

Table 1. Demographic and clinical characteristics of prospectively enrolled patients. ${ }^{A} n=294 ;{ }^{B} n=293$; ${ }^{C} n=262 ;{ }^{D} n=211$. Categorical variables are presented as $n(\%)$ and continuous variables as median $\left(25^{\text {th }}\right.$. $75^{\text {th }}$ percentile). AASs = acute aortic syndrome; AltDs: alternative diagnoses; an. = aneurysm; art. $=$ artery; $\mathrm{BP}=$ blood pressure; dis. $=$ disease; sy. $=$ syndrome; thor. $=$ thoracic.

of transmembrane ST2, is secreted into the circulation and functions as a decoy receptor for interleukin-33. Evidence has accumulated that sST2 is up-regulated in cardiac cells upon myocardial strain and several studies have established plasma sST2 as a biomarker for clinical risk prediction in patients affected by heart failure ${ }^{15-19}$. The role of sST2 within vascular tissues and in the context of aortic disease is largely unknown ${ }^{20}$. However, Wang et al. recently reported that plasma sST2, measured with a research-grade assay, showed striking diagnostic accuracy for AASs and outperformed D-dimer, in a Chinese patient cohort ${ }^{21}$. Since plasma sST2 levels can be measured with a clinical-grade assay already approved for patient use, we sought to further evaluate it as a diagnostic biomarker for AASs in an independent prospective cohort of acute patients from a European ED.

\section{Results}

Study population. In the study period, 297 patients with any pre-defined red-flag symptom (chest/abdominal/back pain, syncope, perfusion deficit) and a clinical suspicion of an AAS, were enrolled. Presenting symptoms were the following: anterior chest pain $(189,63.6 \%)$, posterior chest pain $(114,38.4 \%)$, abdominal pain $(66$, $22.2 \%)$, back pain $(25,8.4 \%)$, syncope $(34,11.4 \%)$, perfusion deficit $(20,6.7 \%)$. The demographic and clinical characteristics of study patients are reported in Table 1 . The prevalence of risk factors for AASs and the corresponding aortic dissection detection risk score are presented in Table 2.243 patients (81.8\%) were classified at low pre-test clinical probability of AAS, according to ESC guidelines ${ }^{13}$.

Diagnostic work-up and case adjudication. The diagnostic workup conducted during the index ED visit included chest X-ray in 166 (55.9\%) patients, focused transthoracic echocardiography in 136 (45.8\%), CTA in $162(54.5 \%)$, transesophageal echocardiography (TEE) in $1(0.3 \%)$ and coronary angiography in $3(1 \%)$. 30-day follow-up data was obtained for 287 (96.6\%) patients. 147 (49.5\%) patients were admitted to hospital. Amongst 10 patients lacking follow-up data, 7 were subjected to advanced aortic imaging in the ED excluding an AAS. Diagnostic data allowing final case adjudication are detailed in Fig. 1.

An AAS was adjudicated in 88 (29.6\%) patients, all subjected to CTA. The AAS types were: 46 (15.5\%) Stanford type A AADs, 20 (6.7\%) Stanford type B AADs, 18 (6.1\%) IMHs, 3 (1\%) SARs and 1 (0.3\%) PAU. In $209(70.4 \%)$ patients, an AAS was excluded and the following alternative diagnoses (AltDs) were adjudicated: skeletal-muscle pain $(45,15.2 \%)$, gastrointestinal disease $(28,9.4 \%)$, acute coronary syndrome $(18,6.1 \%)$, non AAS-related syncope $(16,5.4 \%)$, other aortic disease $(15,5.1 \%)$, pericarditis $(12,4 \%)$, pleurisy/pneumonia $(9$, $3 \%)$, stroke $(1,0.3 \%)$, pulmonary embolism $(1,0.3 \%)$ and other conditions $(64,21.5 \%)$. The demographic and clinical characteristics of study patients classified by diagnostic outcome are reported in Table 1 . Patients affected by AASs had significantly higher levels of white blood cells (WBCs), creatinine, troponin and D-dimer.

CTA-based adjudication. We first analyzed the diagnostic characteristics of plasma sST2 within patients subjected to CTA ( $\mathrm{n}=162,88$ with AASs), which represents the imaging gold standard to rule-in/out AASs. The demographic and clinical characteristics of patients with AltDs subjected to CTA $(n=74)$ are reported in Supplementary Table 1. The median plasma sST2 level was $41.7 \mathrm{ng} / \mathrm{mL}$ (IQR 29.4-103.2) in patients with AASs 


\begin{tabular}{|c|c|c|c|c|}
\hline Variable & Total patients $(n=297)$ & AASs $(\mathbf{n}=88)$ & AltDs $(n=209)$ & $P$-value \\
\hline \multicolumn{5}{|l|}{ History } \\
\hline Marfan/conn. tissue dis. & $1(0.3 \%)$ & $0(0 \%)$ & $1(0.5 \%)$ & 0.52 \\
\hline Family history of AAS & $7(2.4 \%)$ & $2(2.3 \%)$ & $5(2.4 \%)$ & 0.95 \\
\hline Aortic valve disease & $21(7.1 \%)$ & $7(8 \%)$ & $14(6.7 \%)$ & 0.7 \\
\hline Recent aortic manipulation & $3(1 \%)$ & $1(1.1 \%)$ & $2(1 \%)$ & 0.89 \\
\hline Thoracic aortic aneurysm & $40(13.5 \%)$ & $21(23.9 \%)$ & $19(9.1 \%)$ & 0.001 \\
\hline \multicolumn{5}{|l|}{ Pain } \\
\hline Sudden pain & $146(49.2 \%)$ & $55(62.5 \%)$ & $91(43.5 \%)$ & 0.001 \\
\hline Severe pain & $73(24.6 \%)$ & $28(31.8 \%)$ & $45(21.5 \%)$ & 0.003 \\
\hline Ripping/tearing pain & $32(10.8 \%)$ & $20(22.7 \%)$ & $12(5.7 \%)$ & 0.06 \\
\hline \multicolumn{5}{|l|}{ Physical findings } \\
\hline Pulse deficit & $23(7.7 \%)$ & $16(18.2 \%)$ & $7(3.3 \%)$ & $<0.001$ \\
\hline Neurologic deficit & $13(4.4 \%)$ & $7(8 \%)$ & $6(2.9 \%)$ & 0.05 \\
\hline New diastolic murmur & $1(0.3 \%)$ & $1(1.1 \%)$ & $0(0 \%)$ & 0.12 \\
\hline Hypotension & $18(6.1 \%)$ & $15(17 \%)$ & $3(1.4 \%)$ & $<0.001$ \\
\hline \multicolumn{5}{|l|}{ ADD risk score } \\
\hline ADD risk score $=0$ & $88(29.7 \%)$ & $11(12.5 \%)$ & $77(36.8 \%)$ & $<0.001$ \\
\hline ADD risk score $=1$ & $155(52.2 \%)$ & $45(51.1 \%)$ & $110(52.6 \%)$ & 0.81 \\
\hline $\mathrm{ADD}$ risk score $=2-3$ & $54(18.2 \%)$ & $32(36.4 \%)$ & $22(10.5 \%)$ & $<0.001$ \\
\hline
\end{tabular}

Table 2. Aortic dissection detection risk factors and associated risk score of study patients. AASs: acute aortic syndromes; ADD: aortic dissection detection; AltDs: alternative diagnoses; conn.: connective; dis.: disease.

and $34.6 \mathrm{ng} / \mathrm{mL}$ (IQR 21.4-51.5) in patients with AltDs ( $P=0.005$, Fig. 2a). Median sST2 levels were $40.8 \mathrm{ng} / \mathrm{mL}$ (IQR 24-146.8) in type A AADs, $45.7 \mathrm{ng} / \mathrm{mL}$ (IQR 40-79.2) in type B AADs, 35.2 ng/mL in IMHs (IQR 30.1-71.5), $54.2 \mathrm{ng} / \mathrm{mL}$ (IQR $44.7-55.3)$ in SARs and $61.9 \mathrm{ng} / \mathrm{mL}$ in PAUs $(P=0.67$; Fig. 2b). The levels of sST2 in different types of AltDs did not significantly differ $(P=0.34$, Supplementary Fig. 1a).

sST2 levels showed linear correlation with WBC count $(\mathrm{r}=0.3, P<0.001)$, creatinine $(\mathrm{r}=0.19, P=0.018)$ and $\mathrm{D}$-dimer $(\mathrm{r}=0.21, P=0.018)$, but not with troponin $\mathrm{T}(\mathrm{r}=0.14, P=0.1)$, and non-linear correlation with all variables (WBC count, $\rho_{\mathrm{s}}=0.26, P<0001$; creatinine, $\rho_{\mathrm{s}}=0.19, P=0.014$; D-dimer, $\rho_{\mathrm{s}}=0.36, P<0.001$; troponin, $\rho_{\mathrm{s}}=0.25, P=0.04$ ). In patients with AASs, sST2 levels showed linear correlation with WBC count (Fig. 3), and non-linear correlation with WBC count $\left(\rho_{\mathrm{s}}=0.26, P=0.014\right)$, creatinine $\left(\rho_{\mathrm{s}}=0.27, P=0.014\right)$ and $\mathrm{D}$-dimer $\left(\rho_{\mathrm{s}}=0.28, P=0.017\right)$, but not with troponin $\mathrm{T}\left(\rho_{\mathrm{s}}=0.17, P=0.17\right)$. In patients with AltDs, sST2 levels showed linear correlation with WBC count $(\mathrm{r}=0.30 ; P=0.009)$ and $\mathrm{D}$-dimer $(\mathrm{r}=0.26 ; P=0.04)$, but not with creatinine $(\mathrm{r}=0.06 ; P=0.64)$ and troponin $(\mathrm{r}=0.2 ; P=0.1)$; non-linear correlation was found with troponin $\left(\rho_{\mathrm{s}}=0.29\right.$, $P=0.017)$, but not with WBC count $\left(\rho_{\mathrm{s}}=0.09, P=0.46\right)$, creatinine $\left(\rho_{\mathrm{s}}=0.08, P=0.52\right)$ and $\mathrm{D}$-dimer $\left(\rho_{\mathrm{s}}=0.24\right.$, $P=0.06)$.

The receiver operating characteristic (ROC) curves of sST2 and D-dimer for diagnosis of AASs are shown in Fig. 4a,b. The area under the curve (AUC) values of sST2 and D-dimer significantly differed $(P<0.001)$. In patients sampled within 24 hours from symptom onset, the AUC of sST2 ( $\mathrm{n}=118)$ was 0.591 (95\%CI 0.497$0.681)$, while the AUC of D-dimer $(\mathrm{n}=97)$ was $0.842(95 \% \mathrm{CI} 0.753-0.908 ; P<0.001 v s \mathrm{sST} 2)$. In patients at low clinical probability of AAS (as defined by ADD-RS $\leq 1)$, the AUC of sST2 ( $\mathrm{n}=120)$ was 0.661 (95\%CI 0.569 $0.745)$, while the AUC of $\mathrm{D}$-dimer $(\mathrm{n}=98)$ was 0.768 (95\%CI $0.672-0.848 ; P=0.12 v s$ sST2).

Using a conventional cutoff of $500 \mathrm{ng} / \mathrm{mL}$, D-dimer had a sensitivity of $95.8 \%$ (95\%CI $88.1-99.1 \%)$, a specificity of $30.7 \%$ (95\%CI 19.6-43.7\%), a LR + of 1.38 (95\%CI 1.2-1.6) and a LR- of 0.14 (95\%CI 0.04-0.4) for AASs. For sST2, the cutoff maximizing the sensitivity and specificity was $66.5 \mathrm{ng} / \mathrm{mL}$. Table 3 reports the diagnostic test characteristics of sST2 for AASs at different selected cutoffs.

Diagnostic outcome-based adjudication. We next evaluated the diagnostic test characteristics of plasma sST2 in the whole study cohort per prospective enrolment and diagnostic outcome adjudication $(\mathrm{n}=297$, Tables 1 and 2). The median plasma sST2 level was $41.7 \mathrm{ng} / \mathrm{mL}$ (IQR 29.4-103.2) in AASs and 30.1 ng/mL (IQR 20-43.8) in AltDs $(P<0.001$; Fig. 2c). The levels of sST2 in different types of AltDs did not significantly differ $(P=0.44$; Supplementary Fig. 1b).

In patients with AltDs, sST2 showed linear correlation with WBC count $(\mathrm{r}=0.24, P<0.001)$, creatinine $(\mathrm{r}=0.22, P=0.001)$, D-dimer $(\mathrm{r}=0.42, P<0.001)$ and troponin $(\mathrm{r}=0.17, P=0.02)$; non-linear correlation was found with creatinine $(\mathrm{r}=0.16, P=0.025)$, $\mathrm{D}$-dimer $(\mathrm{r}=0.25, P=0.03)$ and troponin $(\mathrm{r}=0.19, P=0.09)$, but not with WBC count $(r=0.05, P=0.43)$. In patients with an AltD of acute coronary syndrome, plasma sST2 levels showed non-linear correlation with troponin T $\left(\rho_{\mathrm{s}}=0.63, P=0.01\right)$, but not with WBC count $\left(\rho_{\mathrm{s}}=0.17, P=0.51\right)$, creatinine $\left(\rho_{\mathrm{s}}=0.32, P=0.22\right)$ and $\mathrm{D}$-dimer $\left(\rho_{\mathrm{s}}=0.04, P=0.89\right)$.

The ROC curves of sST2 and D-dimer for diagnosis of AASs in the full study cohort are shown in Fig. 4c,d. The AUC values of sST2 and D-dimer significantly differed $(P<0.001)$. In patients sampled within 24 hours from symptom onset, the AUC of sST2 $(\mathrm{n}=223)$ was $0.675(95 \%$ CI $0.61-0.736)$, while the AUC of D-dimer $(n=160)$ was 0.917 (95\%CI $0.863-0.955 ; P<0.001 v s$ sST2). In patients at low clinical probability of AAS, the AUC of sST2 
(a) acute aortic syndromes $(n=88)$

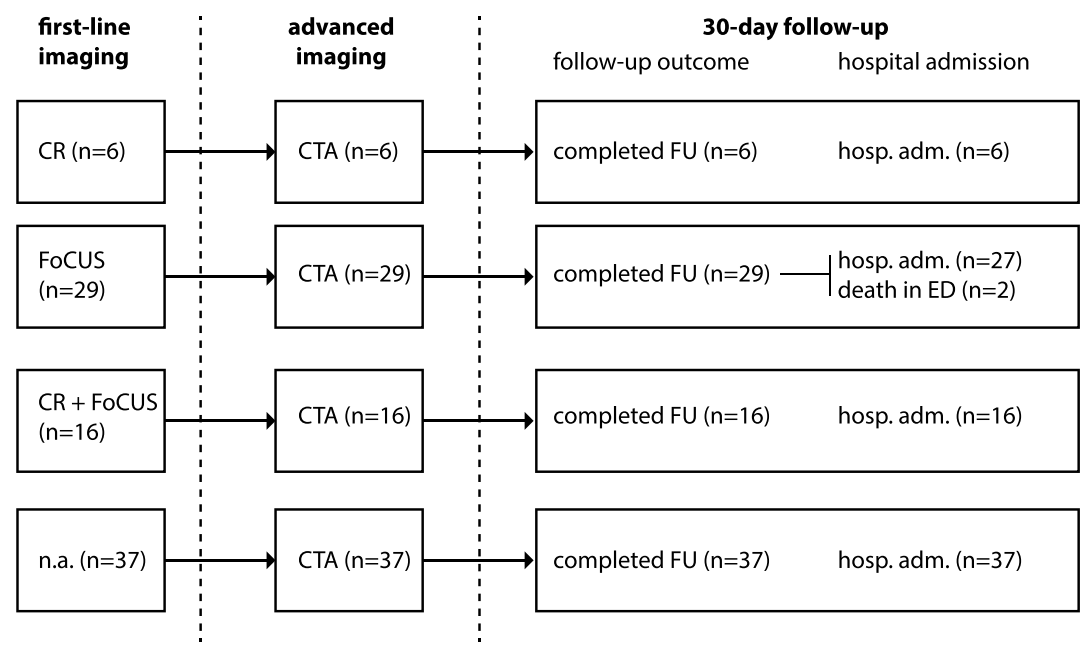

(b) alternative diagnoses $(n=209)$

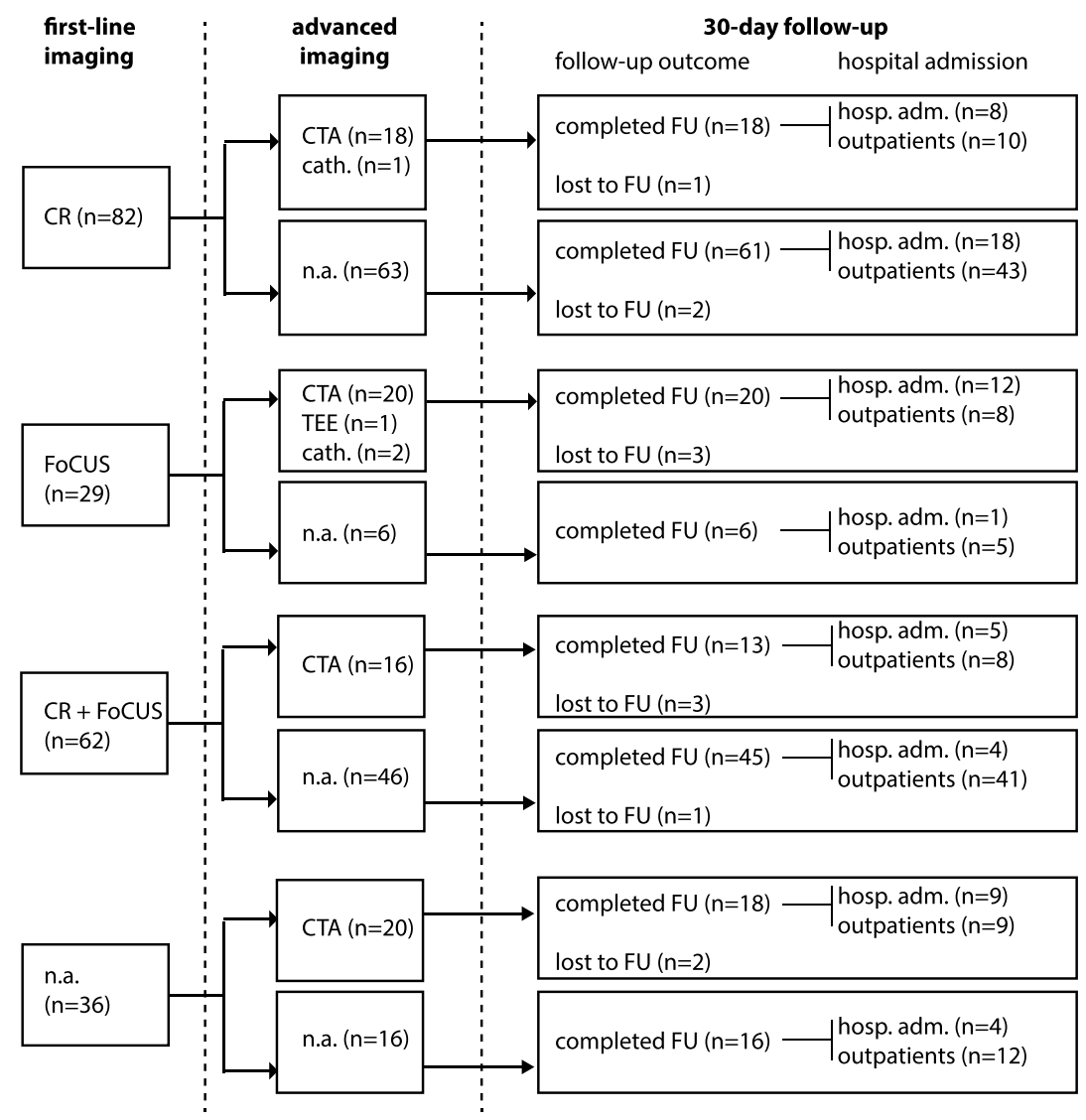

Figure 1. Data used for diagnostic outcome adjudication. Cath: coronary angiography (cardiac catheterization laboratory); CR: chest X-ray; CTA: contrast-enhanced computed tomography angiography of the chest and abdomen; FoCUS: focused cardiac ultrasound; FU: follow-up; hosp. adm.: hospital admission; n.a.: not available; TEE: transesophageal echocardiography.

$(\mathrm{n}=243)$ was 0.717 (95\%CI 0.655-0.772), while the AUC of $\mathrm{D}$-dimer $(\mathrm{n}=168)$ was 0.867 (95\%CI 0.806-0.914; $P=0.002$ vs sST2).

Using a cutoff of $500 \mathrm{ng} / \mathrm{mL}$, D-dimer had a sensitivity of $95.8 \%$ (95\%CI 88.1-99.1\%), a specificity of $53.6 \%$ (95\%CI 45-62\%), a LR + of 2.06 (95\%CI 1.7-2.5) and a LR- of 0.079 (95\%CI 0.03-0.2) for AASs. The diagnostic test characteristics of sST2 for AASs in the full study cohort are reported in Table 3 . The cutoff maximizing the sensitivity and specificity of sST2 for AASs in the full cohort was $39.8 \mathrm{ng} / \mathrm{mL}$. The diagnostic test 
a

Patient adjudication: CTA $(\mathrm{n}=162)$

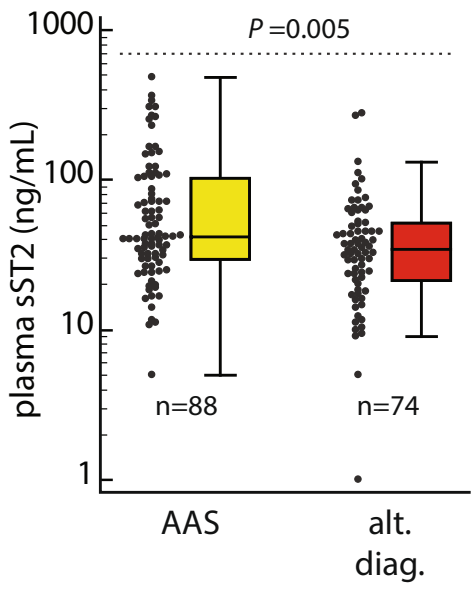

C

Patient adjudication: outcome $(n=297)$

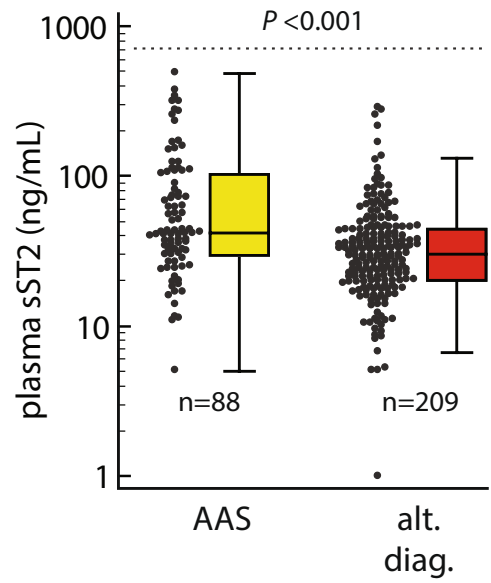

b

Subtypes of acute aortic syndromes $(\mathrm{n}=88)$

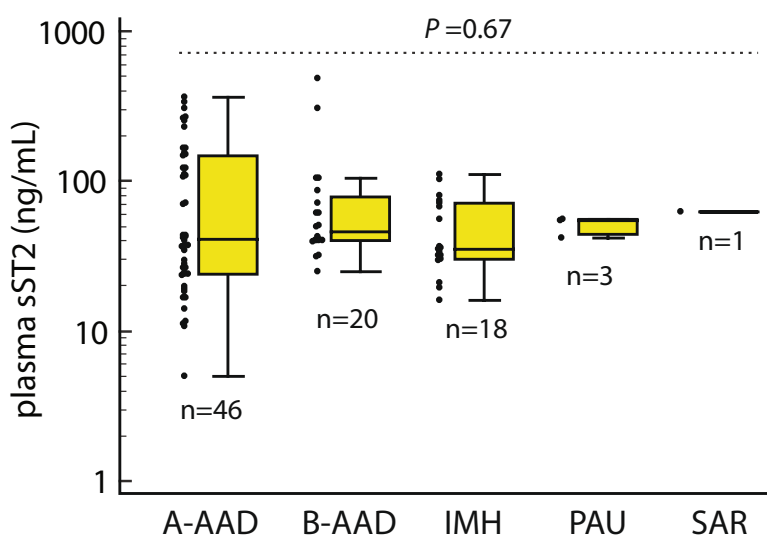

Figure 2. Dot-plot and box-whisker representation of plasma sST2 levels in study patients, classified by: (a) dichotomic final diagnosis (adjudication based on CTA), (b) subtype of acute aortic syndrome, (c) dichotomic final diagnosis (adjudication based on diagnostic outcome). A-AAD: type A acute aortic dissection; AAS: acute aortic syndrome; ACS: acute coronary syndrome; alt. diag.: alternative diagnosis; B-AAD: type B AAD; IMH: intramural aortic hematoma; PAU: penetrating aortic ulcer; SAR: spontaneous aortic rupture.

characteristics associated with sST2 $\geq 39.8 \mathrm{ng} / \mathrm{mL}$ were: sensitivity $58 \%$ (95\%CI $47-68.4 \%)$, specificity $70.8 \%$ (95\% CI 64.1-76.9\%), positive likelihood ratio (LR+) 1.99 (95\% CI 1.5-2.6), negative likelihood ratio (LR-) 0.59 (95\%CI 0.5-0.8).

\section{Discussion}

In this single-center prospective study performed in a European ED, median plasma levels of sST2 measured with a clinical-grade assay were higher in patients with AASs than in patients with AltD. However, there was high inter-patient variability and the overall performance of sST2 for diagnosis AASs was modest. Furthermore, sST2 proved inferior to D-dimer, a well-established biomarker of AASs already applied in clinical practice.

The present study post-dated the study by Wang et al. reporting high sensitivity and specificity of plasma sST2 for AASs and indicating potential superiority of sST2 to D-dimer ${ }^{21}$. Several issues may cause the discrepancy between current and previous data. First, the present study involved a larger case-mix and an elder population, both typical of western country hospitals. Indeed, a recent study has reported significant variation in sST2 levels related to age, with 50 years as a critical cutoff ${ }^{22}$. Major differences in age between the present and the previous study may account for the discrepancy. Second, male gender has also been associated with higher sST2 levels. However, since gender distribution was similar between the two studies, this should not be regarded as a major confounder. Third, ethnic differences in sST2 levels between Asian and European populations may exist. Indeed, the lowest diagnostic accuracy estimates for D-dimer have been obtained in Asian studies ${ }^{23}$. Finally, the present study used a different assay for detection of sST2, i.e. the only one approved for patient use $\mathrm{e}^{24}$. In Wang et al., the 
a

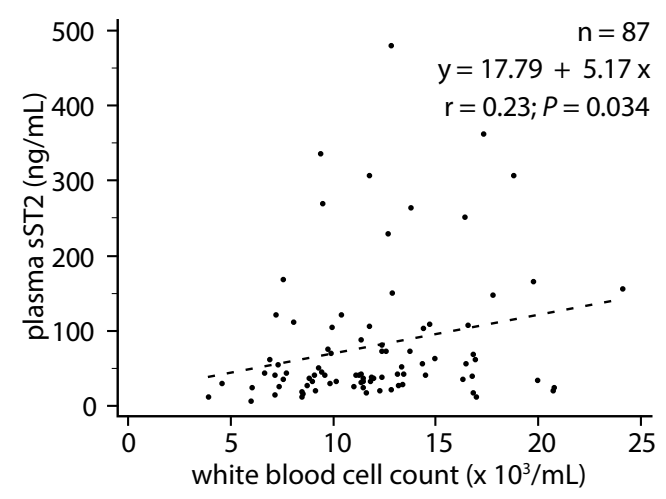

C

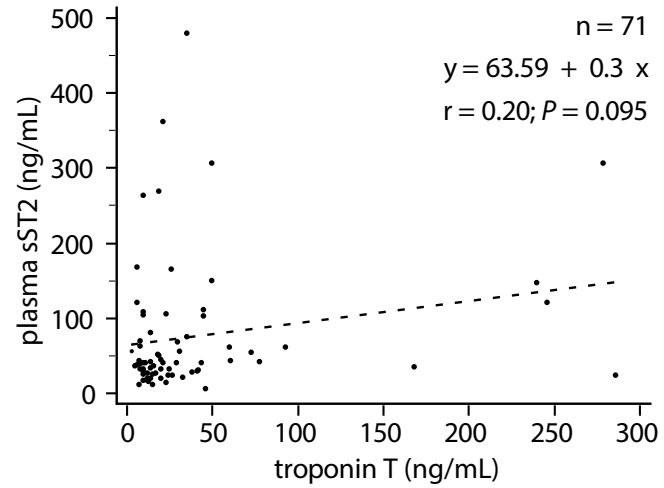

b

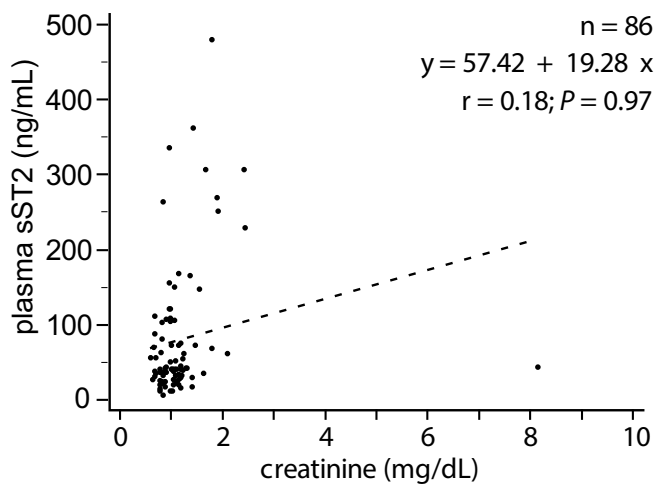

d

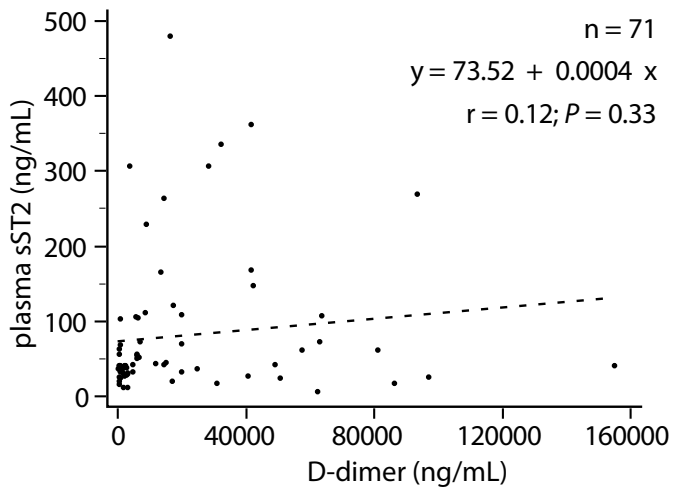

Figure 3. Scatter plots evaluating the correlation and linear regression between plasma sST2 and (a) white blood cell count, (b) creatinine, (c) troponin T and (d) D-dimer, in patients with acute aortic syndromes. Linear regression analysis data are presented as inset.

results of the research-grade R\&D assay and the clinical-grade Presage assay strongly correlated. In the present study, we also found a significant albeit looser correlation between assays. Technical differences between sST2 assays have already been documented ${ }^{25}$. However, additional ethinc specific differences between assays are also conceivable.

Together with previous evidence, current findings indicate that AASs may cause release of sST2 into the bloodstream, but the source and kinetics of this process remain largely unknown. In the present study, the levels of sST2 did not correlate with those of troponin T in AASs, indicating a loose relationship, if any, between plasma sST2 levels and myocardial damage. Instead, the levels of plasma sST2 and troponin T were correlated in patients with AltDs (including subgroup with acute coronary syndromes). In AASs, the levels of plasma sST2 correlated with WBC count and D-dimer, rather suggesting a pathophysiological link with inflammatory pathways engaged by acute aortic lesions ${ }^{8,26,27}$. Also in the study by Wang et al., plasma sST2 positively correlated with D-dimer concentrations and not with those of brain natriuretic peptide.

The present study has limitations. First, clinical suspicion of AASs, representing a key patient selection criterion, falls short of a universal definition. Since the study was performed in a tertiary ED specialized in cardiovascular emergencies, variation of physician's gestalt and local practice might limit result generalizability. Second, due to ethical and clinical considerations (exposure to radiation and contrast medium), CTA could not be performed as uniform gold standard. Treating physicians decided on CTA based on patient-level clinical reasoning irrespective of study participation, in compliance with an observational and non-interventional study protocol. A follow-up approach was used for pragmatic case adjudication based on 30-day outcomes. This method is routinely applied in diagnostic studies of cardiovascular emergencies in the ED such as pulmonary embolism and acute coronary syndromes and has been used in a previous multicenter study of AASs ${ }^{12,28-32}$. As compared to our previous work, the timeline of the follow-up was extended from 14 to 30 days, in order to fully cover both the acute and subacute phases of AASs. Very few patients with clinically mild forms of AASs (e.g. short uncomplicated IMHs or PAUs) might have been misdiagnosed. However, they could not substantially affect the study results. Accordingly, the results obtained in the CTA subgroup and in the full cohort did not differ.

In conclusion, in a European cohort of ED patients, plasma sST2 showed poor diagnostic accuracy for AASs. D-dimer, instead, confirmed its established diagnostic performance characterized by high sensitivity and moderate specificity. Results substantially differ from those previously obtained in a larger and much younger cohort of Chinese patients. Further studies are needed to evaluate sST2 as a potential biomarker of aortic diseases in younger patients of non-Asian ethnicity. 
a

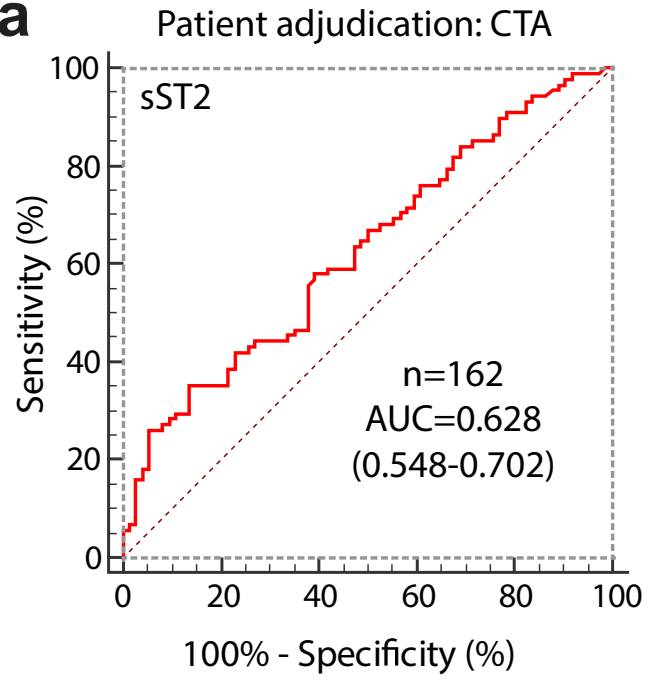

C Patient adjudication: outcome

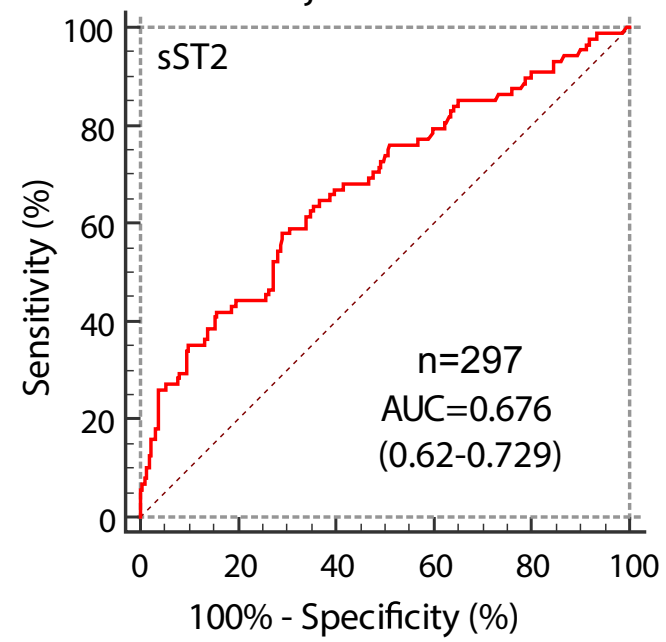

b Patient adjudication: CTA

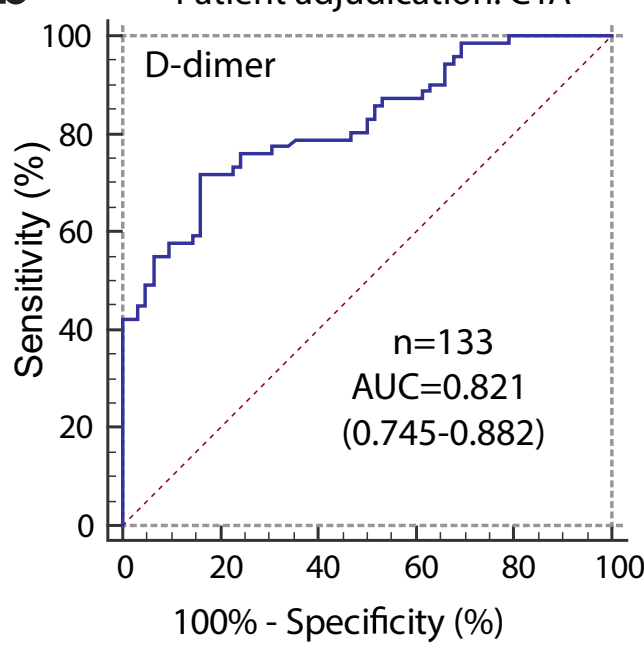

d

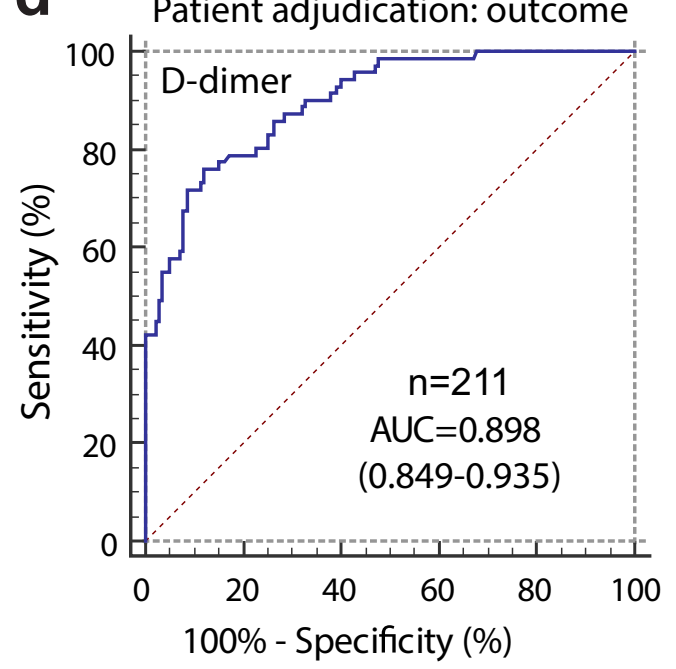

Figure 4. Receiver operating characteristic (ROC) curves of plasma SST2 and D-dimer for diagnosis of acute aortic syndromes in $(\mathbf{a}, \mathbf{b})$ patients subjected to CTA and $(\mathbf{c}, \mathbf{d})$ in all study patients classified according to diagnostic outcome. AUC values are reported with their $95 \% \mathrm{CI}$ in brackets.

\begin{tabular}{|c|c|c|c|c|}
\hline Variable & Case adjudication & $\mathrm{sST} 2 \geq 12 \mathrm{ng} / \mathrm{mL}^{\mathrm{A}}$ & $\mathrm{sST} 2 \geq 23.7 \mathrm{ng} / \mathrm{mL}^{\mathrm{B}}$ & $\mathrm{sST} 2 \geq 66.5 \mathrm{ng} / \mathrm{mL}^{\mathrm{C}}$ \\
\hline Sensitivity (\%) & $\begin{array}{l}\text { CTA }(n=162) \text { and diagnostic } \\
\text { outcome }(n=297)\end{array}$ & $95.5 \%$ (95\%CI 88.8-98.7\%) & $84.1 \%(95 \%$ CI $74.8-91 \%)$ & $35.2 \%(95 \%$ CI $25.3-46.1 \%)$ \\
\hline \multirow{2}{*}{ Specificity (\%) } & CTA $(n=162)$ & $10.8 \%(95 \%$ CI $4.8-20.2 \%)$ & $29.7 \%(95 \%$ CI $19.7-41.5 \%)$ & $85.1 \%(95 \%$ CI $75-92.3 \%)$ \\
\hline & diagnostic outcome $(n=297)$ & $8.6 \%(95 \%$ CI $5.2-13.3 \%)$ & $35.4 \%$ (95\%CI $28.9-42.3 \%)$ & $89.5 \%$ (95\%CI 84.5-93.3\%) \\
\hline \multirow{2}{*}{$\mathrm{LR}+$} & CTA $(n=162)$ & 1.07 (95\%CI 1-1.2) & $1.20(95 \%$ CI 1-1.4) & 2.37 (95\%CI 1.3-4.4) \\
\hline & diagnostic outcome $(n=297)$ & $1.04(95 \%$ CI $1.0-1.1)$ & $1.3(95 \%$ CI $1.1-1.5)$ & 3.35 (95\%CI $2.1-5.4)$ \\
\hline \multirow{2}{*}{ LR- } & CTA $(n=162)$ & $0.42(95 \%$ CI $0.1-1.3)$ & $0.54(95 \%$ CI $0.3-1)$ & $0.76(95 \%$ CI $0.6-0.9)$ \\
\hline & diagnostic outcome $(n=297)$ & 0.53 (95\%CI $0.2-1.5)$ & 0.45 (95\%CI $0.3-0.8)$ & $0.72(95 \%$ CI $0.6-0.9)$ \\
\hline
\end{tabular}

Table 3. Diagnostic test characteristics of sST2 for diagnosis of acute aortic syndromes. ${ }^{A}$ Cutoff providing similar sensitivity to D-dimer (cutoff $500 \mathrm{ng} / \mathrm{mL}$ ) in the CTA-based analysis; ${ }^{B}$ cutoff providing similar specificity to D-dimer (cutoff $500 \mathrm{ng} / \mathrm{mL}$ ) in the CTA-based analysis; ${ }^{{ }}$cutoff maximizing sensitivity and specificity (Youden's method) in the CTA-based analysis. LR: likelihood ratio.

\section{Materials and Methods}

Study design. We conducted a single-center prospective diagnostic accuracy study, in a large urban ED. The methods were carried out in accordance with the relevant guidelines and regulations. The local Human Ethics 
Committee (Comitato Etico Interaziendale A.O.U. Città della Salute e della Scienza di Torino) approved the study and written informed consent was obtained from participants or their authorized representatives. The study protocol conformed to the ethical guidelines (Declaration of Helsinki, 1975).

Selection of participants. From January 2017 to October 2018 , outpatients aged $\geq 18$ years were enrolled: (1) if they presented $\geq 1$ red-flag symptom(s) of AAS (chest/abdominal/back pain, syncope, perfusion deficit) dating $\leq 14$ days, and (2) if an AAS was considered in differential diagnosis by the attending physician. To allow immediate handling and storage of plasma samples in the laboratory, enrolment was limited to daytime, from 8 a.m. to 8 p.m., 7 days/week.

Laboratory analyses. During the ED visit, patients underwent venipuncture for the routine blood tests deemed necessary by the attending physician. Blood samples were immediately sent to the local laboratory for automatic processing. D-dimer levels were measured urgently by the laboratory on trisodium citrate-plasma samples, using the STA-Liatest D-Di assay (Stago, Asnières sur Seine, France). This is a rapid, automated, quantitative immune-turbidimetric quantitative assay. The results of the D-dimer assay were available live to the attending physicians, for clinical decisions. For sST2 measurement, for each patient an EDTA-plasma sample aliquot was rapidly obtained in the laboratory and stored at $-80^{\circ} \mathrm{C}$ until further processing. The levels of sST2 were measured subsequently on thawed plasma batches and were not available to attending physicians for clinical use.

sST2 assay. The concentrations of plasma sST2 were measured using the Critical Diagnostics Presage ${ }^{\circledR}$ ST2, a quantitative sandwich type ELISA (enzyme-linked immunosorbent assay) which uses monoclonal antibodies. Human endogenous ST2 demonstrated stability in all the following conditions: storage at $20^{\circ} \mathrm{C}$ for $48 \mathrm{~h}$, at $4{ }^{\circ} \mathrm{C}$ for 7 days, at $-20^{\circ} \mathrm{C}$ and $-80^{\circ} \mathrm{C}$ for 18 months. The assay precision assessment was performed according to the CLSI (Clinical and Laboratory Standards Institute) EP5-A standards. Intra-series coefficient of variation (CVa) and total CVa were $6.5 \%$ and $9.1 \%$, respectively, at an average concentration of $16.9 \mathrm{ng} / \mathrm{mL}, 3.4 \%$ and $5.5 \%$ at a mean concentration of $33.1 \mathrm{ng} / \mathrm{mL}, 3.8 \%$ and $6.3 \%$ at an average concentration of $68.7 \mathrm{ng} / \mathrm{mL}, 2.4 \%$ and $4.8 \%$ at an average concentration of $159.1 \mathrm{ng} / \mathrm{mL}$. The limit of quantification is $2.4 \mathrm{ng} / \mathrm{mL}$ and the dynamic range of measurement is from $2.4 \mathrm{ng} / \mathrm{mL}$ up to $200 \mathrm{ng} / \mathrm{mL}$. There are no significant interferences with total proteins, triglycerides, hemoglobin, cholesterol, bilirubin.

In a subset of 86 patients ( $24 \mathrm{AAS}, 62 \mathrm{AltD})$ with sufficient plasma aliquots, sST2 levels were assayed also with the human ST2/IL-1 R4s assay by R\&D Systems (DY523B-05; Minneapolis, MN, USA), according to the manufacturer's protocol. Passing and Bablok regression analyses were used for method comparison. The Cusum test showed non-significant deviation from linearity $(\mathrm{p}=0.88)$. Non-parametric correlation analysis revealed a Spearman's coefficient of rank correlation $\left(\rho_{\mathrm{s}}\right)$ of 0.29 (95\%CI, $0.11-0.45 ; P=0.003$ ) between results obtained with the Presage and the $\mathrm{R} \& \mathrm{D}$ assay.

Case adjudication. All diagnostic and clinical decisions were performed at the discretion of the treating providers, independent of study participation. For each patient, a dichotomic case adjudication (AAS $v s$ alternative diagnosis, AltD) was made by two independent reviewers expert in Emergency Medicine and acute aortic syndromes, blinded to sST2 and D-dimer levels, who evaluated all available clinical data (ED/hospital/surgical charts, imaging data, blood test results) obtained within 30 days after the index ED visit, including the results of a 30-day clinical or structured telephone follow-up contact performed by a research staff. In case of discordance, cases were adjudicated after discussion. Patients were instructed to return to the ED in case of new, worsening or recurrent symptoms.

AAS was defined by evidence of an AAS on CTA, TEE, magnetic resonance angiography, surgery or autopsy. If advanced imaging data, surgical or autopsy data was not available, an AAS was considered absent: (1) in patients admitted to hospital, if an alternative diagnosis was available, (2) in patients dismissed from the ED, if they had an uncomplicated clinical course or if an alternative diagnosis was made during the 30-day follow-up period.

Outcome measures and sample size. Primary outcomes were the diagnostic test characteristics of sST2 for AASs. To calculate sample size, we hypothesized to test an AUC difference of $10 \%$ between sST2 and D-dimer, for diagnosis of AASs. Based on metanalysis of available studies, we assumed for D-dimer an AUC of $95 \%{ }^{23}$. Using an alpha of 0.05 (one sided) and a power of 0.8 , we needed to include about 250 patients to reject the null hypothesis.

Data analysis. Continuous variables were expressed as median and IQR (interquartile range, indicated as $25^{\text {th }}$ to $75^{\text {th }}$ percentile values). Dichotomous data were expressed as proportions (in \% value) with their $95 \%$ confident interval (CI), computed using Wilson's method. The diagnostic performance of the biomarkers was evaluated with ROC analysis. AUCs were calculated and compared per Hanley \& McNeil. For specified cutoffs, sensitivity, specificity and positive/negative likelihood ratios (LR+/LR-) were computed with their $95 \% \mathrm{CI}$. The tests used for statistical comparison were Pearson's $\chi^{2}$ test (dichotomous data), Mann-Whitney U-test for independent samples (continuous data, two-groups) and Kruskal-Wallis test for independent samples (continuous data, multiple groups). $P$-values were two-sided and $P<0.05$ was considered significant. The analysis was performed with SPSS statistical package (ver. 25.0, SPSS Inc., Chicago, Illinois) and MedCalc software (ver 18.11.3).

\section{Data availability}

The datasets generated during and analyzed during the current study are available from the corresponding author on reasonable request. 
Received: 13 May 2019; Accepted: 28 January 2020;

Published online: 20 February 2020

\section{References}

1. Bossone, E., LaBounty, T. M. \& Eagle, K. A. Acute aortic syndromes: diagnosis and management, an update. Eur. Heart J. 39, 739-749d, https://doi.org/10.1093/eurheartj/ehx319 (2018).

2. Hansen, M. S., Nogareda, G. J. \& Hutchison, S. J. Frequency of and inappropriate treatment of misdiagnosis of acute aortic dissection. Am. J. Cardiol. 99, 852-856, https://doi.org/10.1016/j.amjcard.2006.10.055 (2007).

3. Zhan, S. et al. Misdiagnosis of aortic dissection: experience of 361 patients. J. Clin. Hypertens. 14, 256-260, https://doi.org/10.1111/ j.1751-7176.2012.00590.x (2012).

4. Pourafkari, L. et al. The frequency of initial misdiagnosis of acute aortic dissection in the emergency department and its impact on outcome. Intern. Emerg. Med. 12, 1185-1195, https://doi.org/10.1007/s11739-016-1530-7 (2017).

5. Kocher, K. E. et al. National trends in use of computed tomography in the emergency department. Ann. Emerg. Med. 58, 452-462 e453, https://doi.org/10.1016/j.annemergmed.2011.05.020 (2011).

6. Lovy, A. J., Bellin, E., Levsky, J. M., Esses, D. \& Haramati, L. B. Preliminary development of a clinical decision rule for acute aortic syndromes. Am. J. Emerg. Med. 31, 1546-1550, https://doi.org/10.1016/j.ajem.2013.06.005 (2013).

7. Ohle, R., Anjum, O., Bleeker, H., Wells, G. \& Perry, J. J. Variation in emergency department use of computed tomography for investigation of acute aortic dissection. Emerg Radiol, https://doi.org/10.1007/s10140-018-1587-x (2018).

8. Morello, F., Piler, P., Novak, M. \& Kruzliak, P. Biomarkers for diagnosis and prognostic stratification of aortic dissection: challenges and perspectives. Biomark Med. 8, 931-941, https://doi.org/10.2217/bmm.14.38 (2014).

9. Suzuki, T. \& Eagle, K. A. Biomarker-Assisted Diagnosis of Acute Aortic Dissection. Circulation 137, 270-272, https://doi. org/10.1161/CIRCULATIONAHA.117.032048 (2018).

10. Suzuki, T. et al. Biomarkers of aortic diseases. Am. Heart J. 165, 15-25, https://doi.org/10.1016/j.ahj.2012.10.006 (2013).

11. Asha, S. E. \& Miers, J. W. A Systematic Review and Meta-analysis of D-dimer as a Rule-out Test for Suspected Acute Aortic Dissection. Ann. Emerg. Med. 66, 368-378, https://doi.org/10.1016/j.annemergmed.2015.02.013 (2015).

12. Nazerian, P. et al. Diagnostic Accuracy of the Aortic Dissection Detection Risk Score Plus D-Dimer for Acute Aortic Syndromes: The ADvISED Prospective Multicenter Study. Circulation 137, 250-258, https://doi.org/10.1161/CIRCULATIONAHA.117.029457 (2018).

13. Erbel, R. et al. 2014 ESC Guidelines on the diagnosis and treatment of aortic diseases: Document covering acute and chronic aortic diseases of the thoracic and abdominal aorta of the adult. The Task Force for the Diagnosis and Treatment of Aortic Diseases of the European Society of Cardiology (ESC). Eur. Heart J. 35, 2873-2926, https://doi.org/10.1093/eurheartj/ehu281 (2014).

14. Griesenauer, B. \& Paczesny, S. The ST2/IL-33 Axis in Immune Cells during Inflammatory Diseases. Front. Immunol. 8, 475, https:// doi.org/10.3389/fimmu.2017.00475 (2017).

15. Weinberg, E. O. et al. Expression and regulation of ST2, an interleukin-1 receptor family member, in cardiomyocytes and myocardial infarction. Circulation 106, 2961-2966 (2002).

16. Pascual-Figal, D. A. et al. Clinical relevance of sST2 in cardiac diseases. Clin. Chem. Lab. Med. 54, 29-35, https://doi.org/10.1515/ cclm-2015-0074 (2016).

17. Aimo, A. et al. Meta-Analysis of Soluble Suppression of Tumorigenicity-2 and Prognosis in Acute Heart Failure. JACC Heart Fail. 5 , 287-296, https://doi.org/10.1016/j.jchf.2016.12.016 (2017).

18. Emdin, M. et al. sST2 Predicts Outcome in Chronic Heart Failure Beyond NT-proBNP and High-Sensitivity Troponin T. J. Am. Coll. Cardiol. 72, 2309-2320, https://doi.org/10.1016/j.jacc.2018.08.2165 (2018).

19. Bayes-Genis, A., Nunez, J. \& Lupon, J. Soluble ST2 for Prognosis and Monitoring in Heart Failure: The New Gold Standard? J. Am. Coll. Cardiol. 70, 2389-2392, https://doi.org/10.1016/j.jacc.2017.09.031 (2017).

20. Martinez-Martinez, E. et al. A role for soluble ST2 in vascular remodeling associated with obesity in rats. PLoS One 8, e79176, https://doi.org/10.1371/journal.pone.0079176 (2013).

21. Wang, Y. et al. Magnitude of Soluble ST2 as a Novel Biomarker for Acute Aortic Dissection. Circulation 137, 259-269, https://doi. org/10.1161/CIRCULATIONAHA.117.030469 (2018).

22. Coronado, M. J. et al. Elevated Sera SST2 Is Associated With Heart Failure in Men $</=50$ Years Old With Myocarditis. J. Am. Heart Assoc. 8, e008968, https://doi.org/10.1161/JAHA.118.008968 (2019).

23. Watanabe, H. et al. Diagnostic test accuracy of D-dimer for acute aortic syndrome: systematic review and meta-analysis of 22 studies with 5000 subjects. Sci. Rep. 6, 26893, https://doi.org/10.1038/srep26893 (2016).

24. Mueller, T. \& Dieplinger, B. The Presage((R)) ST2 Assay: analytical considerations and clinical applications for a high-sensitivity assay for measurement of soluble ST2. Expert. Rev. Mol. Diagn. 13, 13-30, https://doi.org/10.1586/erm.12.128 (2013).

25. Mueller, T., Zimmermann, M., Dieplinger, B., Ankersmit, H. J. \& Haltmayer, M. Comparison of plasma concentrations of soluble ST2 measured by three different commercially available assays: the MBL ST2 assay, the Presage ST2 assay, and the R\&D ST2 assay. Clin. Chim. Acta 413, 1493-1494, https://doi.org/10.1016/j.cca.2012.06.021 (2012).

26. Wen, D. et al. Plasma concentrations of interleukin-6, C-reactive protein, tumor necrosis factor- $\alpha$ and matrix metalloproteinase-9 in aortic dissection. Clin. Chim. Acta 413, 198-202, https://doi.org/10.1016/j.cca.2011.09.029 (2012).

27. Morello, F. et al. White blood cell and platelet count as adjuncts to standard clinical evaluation for risk assessment in patients at low probability of acute aortic syndrome. Eur. Heart J. Acute Cardiovasc. Care 6, 389-395, https://doi.org/10.1177/2048872615600097 (2017).

28. Morello, F. et al. Prospective diagnostic and prognostic study of copeptin in suspected acute aortic syndromes. Sci. Rep. 8, 16713, https://doi.org/10.1038/s41598-018-35016-z (2018).

29. Nazerian, P. et al. Integrated use of conventional chest radiography cannot rule out acute aortic syndromes in emergency department patients at low clinical probability. Acad. Emerg. Med. https://doi.org/10.1111/acem.13819 (2019).

30. Nazerian, P. et al. Integration of transthoracic focused cardiac ultrasound in the diagnostic algorithm for suspected acute aortic syndromes. Eur. Heart J. 40, 1952-1960, https://doi.org/10.1093/eurheartj/ehz207 (2019).

31. Twerenbold, R. et al. Outcome of Applying the ESC 0/1-hour Algorithm in Patients With Suspected Myocardial Infarction. J. Am. Coll. Cardiol. 74, 483-494, https://doi.org/10.1016/j.jacc.2019.05.046 (2019).

32. Kearon, C. et al. Diagnosis of Pulmonary Embolism with d-Dimer Adjusted to Clinical Probability. N. Engl. J. Med. 381, 2125-2134, https://doi.org/10.1056/NEJMoa1909159 (2019).

\section{Author contributions}

F.M. conceived and designed the study; F.M., A.B., M.B., M.S. acquired clinical data; A.I., F.R., G.M. performed technical assays and analyzed biochemical data; F.M., M.S. and E.P. conducted the statistical analysis; F.M. and E.L. analyzed and interpreted the data; F.M., A.B. and M.B. wrote the article; all the authors participated in manuscript review and revision and provided final approval of the version to be published. All authors had full access to all the data in the study, take responsibility for the integrity of the data and the accuracy of the data analysis. 


\section{Competing interests}

F.M. reports grant money from the Italian Ministry of Health (GR-2013-02355449, unrelated to the present work), and honoraria from Boehringer Ingelheim and Bayer for lectures and educational activities unrelated to the present work. The other authors report no conflicts of interest.

\section{Additional information}

Supplementary information is available for this paper at https://doi.org/10.1038/s41598-020-59884-6.

Correspondence and requests for materials should be addressed to F.M.

Reprints and permissions information is available at www.nature.com/reprints.

Publisher's note Springer Nature remains neutral with regard to jurisdictional claims in published maps and institutional affiliations.

(c) (i) Open Access This article is licensed under a Creative Commons Attribution 4.0 International License, which permits use, sharing, adaptation, distribution and reproduction in any medium or format, as long as you give appropriate credit to the original author(s) and the source, provide a link to the Creative Commons license, and indicate if changes were made. The images or other third party material in this article are included in the article's Creative Commons license, unless indicated otherwise in a credit line to the material. If material is not included in the article's Creative Commons license and your intended use is not permitted by statutory regulation or exceeds the permitted use, you will need to obtain permission directly from the copyright holder. To view a copy of this license, visit http://creativecommons.org/licenses/by/4.0/.

(C) The Author(s) 2020 